\title{
Development the algorithm of positioning industrial wares in-plant based on radio frequency identification for the products tracking systems
}

\author{
A.V. Astafiev ${ }^{1}$, A.A. Orlov ${ }^{1}$, D.P. Popov ${ }^{1}$ \\ ${ }^{1}$ Murom Institute of Vladimir State University, Orlovskaya, 23, 602264, Murom, Russia
}

\begin{abstract}
As the title implies the article describes actuality of algorithm development of positioning industrial wares in-plant based on radio frequency grid for the construction of the products tracking systems. Requirements of international standards regulating the processes of traceability and identification are analysed. The article offers a system hardware solution for positioning of industrial wares in-plant based on radio frequency grid as well as an algorithm for determining the current storage area. Experimental studies of the developed algorithm were conducted.
\end{abstract}

Keywords: positioningж traceabilityж radio frequency identificationж RFID

\section{Introduction}

Is the identification mechanism which provides the traceability of products during whole technological cycle of production. According to MN ISO 9001-87 requirements a supplier, if necessary, must set and support a method of product identification on all stages of production [1]. Traceability in production helps to provide compliance of requirements of government and international standards of quality, execute a rapid and targeted track of products during the technological cycle that, in turn, allows minimizing financial consequences. Especially important the question of tracking of products becomes if the technological cycle consists of large number of stages located on large territorial areas.

In the last few years instead of graphic marking and systems of technical vision systems companies prefer to use the radio frequency identification method. Currently radio frequency identification is one of the best information technologies used for constructing inventory control systems. Radio frequency identification is used for accounting tasks in different areas of activity, for example in logistics, libraries, shops, etc. However, the task of development and deployment of a complete system for product tracking in production still remains unsolved. Based on this one can conclude that development of new algorithms for identification and positioning of industrial wares in-plant based on radio frequency grid for the construction of the systems of product tracking is an actual scientific and technical task.

\section{Setting the production requirements for the process of radio frequency identification in the products tracking systems}

Let us consider base concepts of this area. Radio frequency identification [radio frequency identification; RFID] is technology of automatic identification and capture of data that uses electromagnetic or inductive connection carried out by means of radio waves for interaction with a radio frequency mark and an unambiguous read-out of its identification data by applying different types of signal modulation and data encoding. Interrogation - interaction of reader / survey device with an RFID tag to read data from it. Backscatter - the process that an RF tag uses to respond to the signal and to react to the electromagnetic field of a reading / interrogation device by modulating and re-radiating it, without changing the carrier frequency. [2]

As the operating frequency of the RFID-tag and the system there are the following ranges: low frequency (LF) - 125-134 $\mathrm{kHz}$, high frequency (HF) - $13.56 \mathrm{MHz}$, ultra high frequency (UHF) - 860-960 MHz , microwave (SHF) - $2.4 \mathrm{GHz}$. Accordingly, for each range there is a corresponding standard, which specifies requirements for it. For example, the general requirements for the air interface for on 860-960 MHz frequency band can be found in the standard [3].

There are a number of standards which establish the structure of RFID tags. Standard [4] reviews unique radio frequency tags that are used for the purposes of: quality control of integrated circuits, which are used in RF tag manufacturing process; RFID traceability during their production and during their term of service; completing the process for reading information of RFID system configuration, including multiple antennas; implementation of anti-collision algorithm for inventory plurality of RFID tags, while in the zone of a reading / interrogation device; traceability of the object with the RF tag on. The standard [5] lists the requirements for the selection of RFID tags, as well as other data carriers, adhesive, face of the label material and ink. This standard specifies methods for reducing the influence of electrostatic discharge and damage to the RFID tag, as well as methods of data verification of the RFID tag. The Standard lists RFID placement and attachment rules.

It is important to point out that RFID-technologies can be subject to an attack. The most common attacks are: RFID-Zapper, cloning, Dos-attacks, attack via other RFID-tags, substitution of RFID-tags memory contents. For the protection of RFID systems experts give the following recommendations: while creating new software publish the code to third-party developers who for a fee can help find bugs, admitted in the development, and remove unnecessary functions [5].

During the system analysis of interstate and international standards requirements to the process of RF identification of products were established. Thus, to develop a radio frequency identification algorithm we must:

1. Define the task of identifying and selecting the appropriate method.

2. Develop a model and to consider all the requirements for it.

3. Determine which keywords should be used, their parameters and the range of operating frequencies. 


\section{Subject overview}

A great contribution to the development of radio frequency identification technology and product movement control systems in various spheres of life was made by Bondarevsky A.S., Zolotov R.V., Do Zuy Nyat, Kamozin D.U., Manish B., Shahram M., Ke-Sheng Wang, Worapot Jakkhupan, Somjit Arch-int, Yuefeng Li, Mahir Oner, Alp Ustundag, Aysenur Budak and many others.

Application of these knowledge-intensive technologies makes it possible to automate the processes of controlling the product movement at industrial plants, ultimately, to increase the efficiency and reliability of transportation control and warehouse inventory control of manufactured products.

However, they are not without flaws. The use of existing software and hardware solutions is more aimed at organizing automated warehouse inventory control and less suitable for automating product movement control, in the absence of universal methods and algorithms. In support of this, at a number of industrial enterprises, developers of RFID systems attempted to organize traceability of products by automatic movement control based on radio frequency identification. As a result, it became clear that automatic control of the product movement is possible only in certain areas of the production process. Such areas are conveyor lines and transport tunnels, where the transportation of products is carried out along the permanently installed radio frequency identification equipment (RFID tunnels). In other production and warehouse areas, automatic control over the movement of products is impossible. This is due to the lack of universal methods and algorithms for product identification in the process of its transportation along unmapped routes.

Positioning of objects and people using information technology is quite a substantial task. These technologies can be used to solve social, industrial and other types of tasks. Currently, there are a large number of approaches to positioning using a large number of technologies, among them:

- Satellite navigation technologies (GPS, GLONASS);

- Local positioning technology (infrared and ultrasonic);

- Technology of technical vision;

- Radio-frequency technologies.

The use of satellite navigation technology and positioning are tightly integrated into our daily lives. They are used for navigation and transport tracking, monitoring and coordination of various kinds of events. The accuracy of positioning is 10-15 meters outdoors. Unfortunately, the application of this technology inside production facilities is almost impossible. An exception is the installation of expensive equipment for organizing GPS-positioning indoors, the unit of which can cover no more than 10 square meters, which is unacceptable for most industrial plants, whose sizes can be tens of kilometers.

Local positioning technologies are highly accurate - about 2 centimeters, but with a short range of 5-10 meters. With these attributes, they are used to achieve local accurate results and, in general, are used for flaw detection (analysis of welds, detection of chips, dents, etc.). The use of local positioning technology for small-scale mechanization is not economically effective and will lead to huge financial costs.

The use of vision technology for solving positioning problems is a relatively young concept. Currently, there are a huge number of methods and algorithms for solving localization and positioning problems, but their effectiveness depends a lot on meeting a large number of requirements, which include the quality of materials used for production of visual labels, cleanliness and lighting of premises, staff attentiveness, etc. Failure to comply with even one of the requirements can lead to a significant reduction in positioning accuracy or make it completely inoperative.

Radio-frequency technologies have found wide application in sales (organization of security in stores). Positioning based on radio frequency technologies can be divided into two categories: positioning on passive RFID tags (distance up to 5 meters) and active RFID beacons (distance up to 80 meters), but all of them are based on the principle that the moved object is marked with an RFID tag and the reading equipment is stationary. This approach allows to effectively automate production processes, where the product movement routes are strictly limited, for example, conveyor lines. However, for the positioning of chaotically moving small mechanization means, this approach will lead to a significant increase in the cost of the positioning system. Instead of a few readers they would need ten times as many.

Considering all the information stated above it is possible to draw a conclusion, that development of technology and software for the construction of positioning and control systems for small mechanization in industrial plants based on radio frequency identification methods is a substantial scientific and technical task.

The development of software and hardware for movement control systems is carried out by: PCT-Invent (Russia, SaintPetersburg), AiTiProject (Russia, Moscow), Impinj (USA, Seattle), Motorola (USA, Morrisville), Nordic ID (Finland, Salo), FEIG (Germany, Weilburg).

Development of positioning systems based on radio frequency identification is carried out by the following scientific organizations:

- Human positioning systems, in particular patients in medical institutions: Shonan Institute of Technology (Japan, Fujisawa), Institute of Medicine (Kathmandu, Nepal), National Patient Safety Foundation (USA, Boston) and others.

- Systems for positioning moving non-metallic objects: East China Jiaotong University (China, Nanchang), Universiti Sains Malaysia (Malaysia, Nibong Tebal), University of Adelaide (Australia, Adelaide), Wellness Convergence Research Center (Korea, Daegu) and many others. 
However, the tasks of developing and implementing automatic systems for tracking products in production are still unresolved. Currently, industrial enterprises still have a number of problems, the solution of which is not realized with the help of modern product movement control systems.

\section{Development of the project hardware positioning system for wares in-plant based on the basis of radio-frequency grid}

As the basic data for the implementation of functional tracking of industrial products arises in the course of its movement through the territory of the plant, it is advisable to develop a hardware solution for receiving and processing of the data. The main types of traffic information are the information about who moves the products, how, departure point and point of arrival. Receiving and processing of this information will allow to organize a permanent automatic traceability of industrial products in the plant.

The paper proposes the development of a stand-alone device, consisting of reading equipment, processing and transmission of information. The developed device is mounted on the transport device, and to ensure that the product gets delivered, plant territory is marked with RFID tags creating a radio frequency grid.

Thus, the hardware part of the system can be divided into 5 levels:

1. The RF tag for labeling storage areas.

2. Equipment to read RFID tags.

3. Equipment for collecting and processing statistical data.

4. The equipment for the transmission of data to the enterprise server.

5. Company's software and hardware.

Laboratory prototype was developed for testing the project hardware industrial products positioning system on the territory of the enterprise on the basis of radio-frequency grid for experimental studies. Laboratory prototype consists of a microcontroller, a manual RF reader, power supply unit and a laptop (Figure 1).

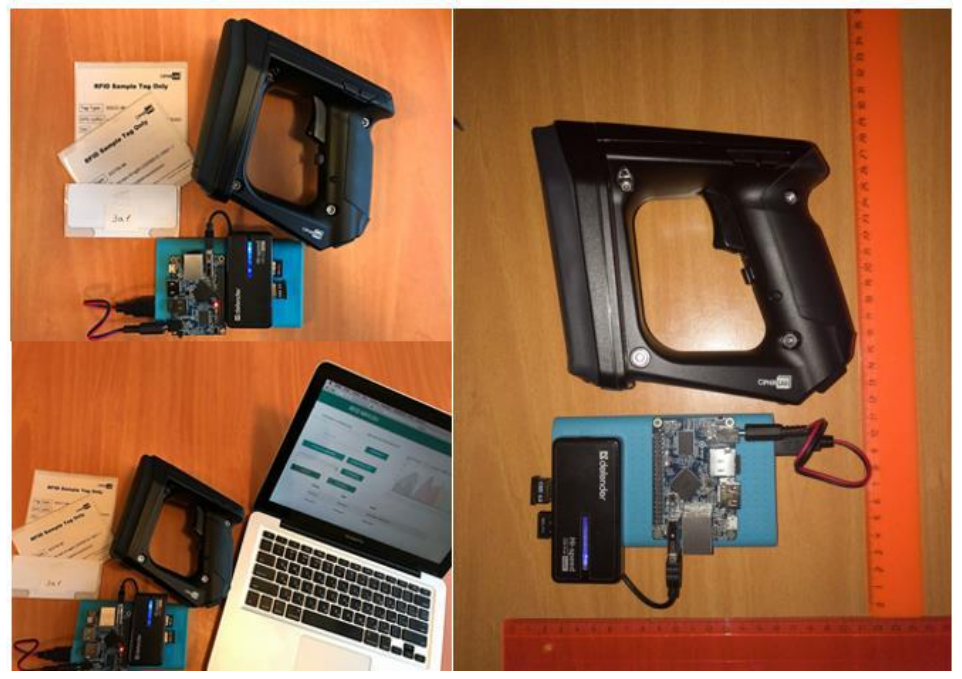

Fig. 1. Type of laboratory prototype.

\section{The algorithm for determining the current storage area}

In order to determine the current position of the transport device for continuous automatic monitoring of transported goods has been developed an algorithm to determine the current storage area.

The algorithm is based on statistical analysis of the number of recognitions of radio frequency tags for certain time periods $t$. The period of time $t$ is the average period of time during which the transport device is moved from the beginning of the current scan area to the end (Figure 2).

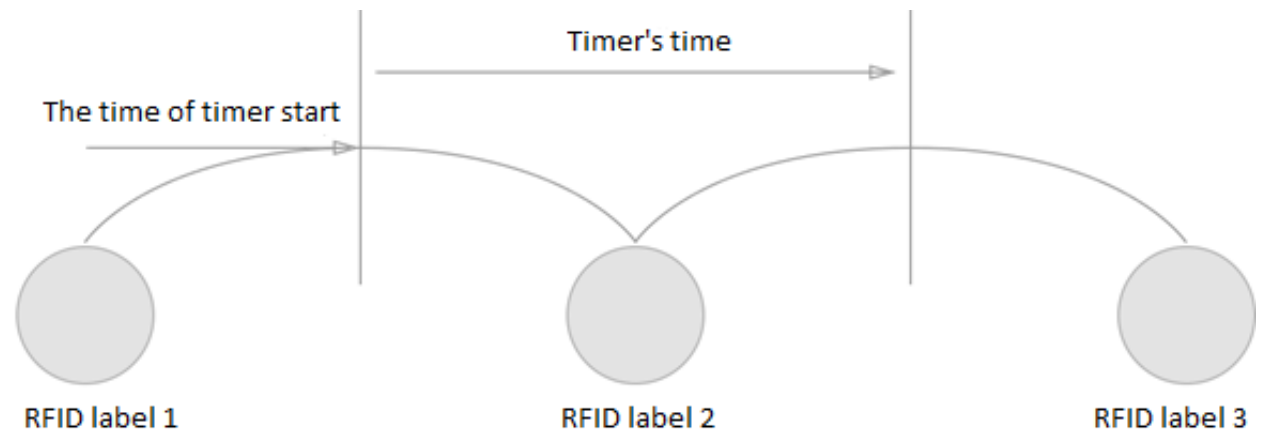

Fig. 2. Is a chart of determination of temporal interval of $t$.

Let the identifiers of warehousing zones be presented as a vector of I : 


$$
\mathrm{I}=\left(\mathrm{I}_{1}, \mathrm{I}_{2}, \mathrm{I}_{3}, . ., \mathrm{I}_{\mathrm{n}}\right)
$$

The amount of recognitions of radio frequency identifiers for the moment of time of $t$ is presented in a kind:

$$
\mathrm{C}=\left(\mathrm{C}_{1}(\mathrm{t}), \mathrm{C}_{2}(\mathrm{t}), \mathrm{C}_{3}(\mathrm{t}), . ., \mathrm{C}_{\mathrm{n}}(\mathrm{t})\right)
$$

Then determination of current position takes place by the calculation of index of $\mathrm{k}$ using a formula:

$$
C_{k}(t)=\left\{\begin{array}{l}
\max _{i} C_{i}(t), \text { if } C_{i}(t)>p \\
\text { undefined, otherwise }
\end{array}\right.
$$

$\mathrm{Ck}(\mathrm{t})$ is the maximal amount of recognitions of radio frequency $\mathrm{k}$-th identifier. It means that in current moment " $\mathrm{t}$ " a transporting device is above the zone of warehousing market " $\mathrm{k}$ ".

\section{The experimental results of algorithm to determine the current storage area}

During the experimental studies many different types of situations that are close to production were modeled (Figure 3). Among them:

- movement between two storage areas;

- move between three or more storage areas;

- movement between storage areas with the presence of "noise" (the other RFID tags, which are not labeled storage areas)

- movement between storage areas with partial overlap with non-metallic and metallic barriers.

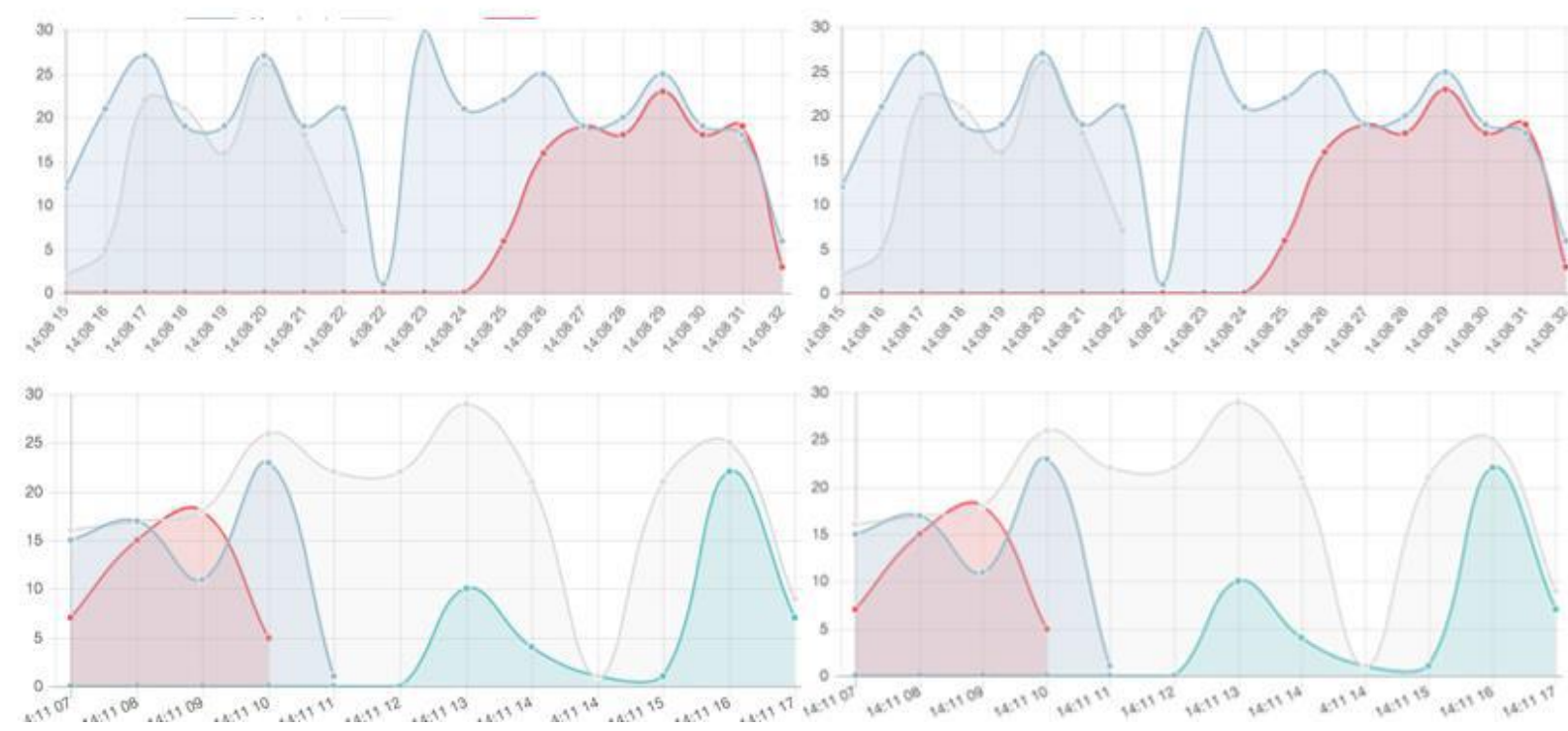

Fig. 3. Results of experimental studies.

Experimental studies have shown the correctness of the algorithm to determine the current storage area in the laboratory.

\section{Conclusion}

The article showed relevance of developing an algorithm of positioning industrial wares in-plant based on radio frequency grid for to create the products tracking systems. We analysed the requirements of international standards regulating the processes of traceability and identification. The article offered a system hardware solution for positioning of industrial wares in-plant based on radio frequency grid as well as an algorithm for determining the current storage area. Experimental studies of the developed algorithm were conducted.

\section{Acknowledgements}

Acknowledgements and Reference heading should be left justified, bold, with the first letter capitalized but have no numbers. Text below continues as normal.

\section{References}

[1] ISO 8402:1994 Quality management and quality assurance - Vocabulary. URL: http://www.docload.ru/Basesdoc/5/5812/index.htm .

[2] GOST R ISO/IEC 19762-3-2011 Information technology. Technology automatic identification and data collection (AISD). The harmonized dictionary. Part 3. radio frequency identification (RFID), 2012-05-01; $20 \mathrm{p}$.

[3] GOST R ISO/IEC 18000-6-2013 Information technology. Radio frequency identification for control items. Part 6. The parameters of the radio interface for the frequency range 860 - $960 \mathrm{MHz}$. General requirements, 2014-01-01; $20 \mathrm{p}$.

[4] GOST R ISO/IEC 15963-2011 Information technology. Radio frequency identification for control items. Unique identification of RFID tags, 2012-01-01; 28 p. 
Image Processing, Geoinformation Technology and Information Security / A.V. Astafiev, A.A. Orlov, D.P. Popov

[5] GOST R 54621-2011 Information technology. Radio frequency identification for control items. Recommendations for use. Part 1. Labels and packaging radio-frequency tag according to ISO/IEC 18000-6 (type C), 2012-06-01; 71p.

[6] Provotorov A, Privezentsev D, Astafiev A. Development of Methods for Determining the Locations of Large Industrial Goods During Transportation on the Basis of RFID. Procedia Engineering 2015; 129: 1005-1009. DOI: 10.1016/j.proeng.2015.12.163.

[7] Astafiev AV, Orlov AA, Provotorov AV. The method of combining the results of localization algorithms for character and bar code labels. Stability and Control Processes in Memory of V.I. Zubov (SCP). International Conference, St. Petersburg, 5-9 Oct. 2015: 618-619. DOI: 10.1109/SCP.2015.7342240.

[8] Astafiev AV, Orlov AA, Provotorov AV. The localization algorithm of symbolic and bar-code labels on industrial products for the control of product movements. Stability and Control Processes in Memory of V.I. Zubov (SCP). International Conference, St. Petersburg, 5-9 Oct. 2015: 615-616. DOI: 10.1109/SCP.2015.7342230

[9] Orlov A, Astafiev A. Development of algorithm for localization of production markings with the use of analysis of the color data on digital images. Geoconference on informatics, geoinformatics and remote sensing conference proceedings. Albena, Bulgaria 2014; 1: 113-118.

[10] Orlov AA, Antonov LV, Astaf'ev AV. Development and experimental investigation of algorithms for distinguishing fuzzy boundaries of objects in photographs of industrial materials. Pattern Recognition and Image Analysis 2015; 25 (3): 509-513.

[11] Terekhin AV. The Algorithm for Generating Pairs of Projections of Three-Dimensional Objects on Two Images. J. Applied Mechanics and Materials 2015; 770: 604-607.

[12] Antonov LV, Orlov AA. Document Research and development of algorithms for objects detection on images of industrial materials. Proceedings of 2014 International Conference on Mechanical Engineering, Automation and Control Systems, MEACS 2014.

[13] Muhammad Shahzad, Liu AX. Identification of Active RFID Tags with Statistically Guaranteed Fairness. IEEE 23rd International Conference on Network Protocols (ICNP) 2015: 279-290. DOI: 10.1109/ICNP.2015.23.

[14] Muhammad Shahzad, Liu AX. Probabilistic Optimal Tree Hopping for RFID Identification. IEEE/ACM Transactions on Networking 2015: 796-809. DOI: 10.1109/TNET.2014.2308873. 